\title{
Enhanced Experimental Tumor Metastasis with Age in Senescence- Accelerated Mouse
}

\author{
Kosuke Shimizu, ${ }^{a}$ Naomi Kinouchi Shimizu, ${ }^{a}$ Tomohiro Asai, ${ }^{a}$ Hideo TsukadA, ${ }^{b}$ and Naoto OkU $*, a$ \\ ${ }^{a}$ Department of Medical Biochemistry and Global COE Program, School of Pharmaceutical Sciences, University of \\ Shizuoka; 52-1 Yada, Suruga-ku, Shizuoka, Shizuoka 422-8526, Japan: and ${ }^{b}$ Central Research Laboratory, Hamamatsu \\ Photonics K.K.; 5000 Hirakuchi, Hamakita-ku, Hamamatsu, Shizuoka 434-8601, Japan. \\ Received December 27, 2007; accepted January 20, 2008; published online February 29, 2008
}

Tumor metastasis is affected by the host immune surveillance system. Since aging may attenuate the host immune potential, the experimental tumor metastasis may be enhanced with age. In the present study, we investigated this alteration of experimental tumor metastasis with age. We used senescence-accelerated mice prone 10 (SAMP10) as a model of aged animals. Natural killer cell (NK) activity, as an indicator of immune surveillance potential, in 8-month-old (aged) SAMP10 mice was observed to be much lower than that in 2-month-old (young) mice. When we examined the in vivo trafficking of lung-metastatic K1735M2 melanoma cells in SAMP10 with positron emission tomography (PET), K1735M2 cells labeled with $\left[2-{ }^{18} \mathrm{~F}\right] 2$-deoxy-2-fluoro-D-glucose $\left(\left[{ }^{18} \mathrm{~F}\right] \mathrm{FDG}\right)$ were observed in both young and aged SAMP10 just after injection of the cells, whereas the clearance of ${ }^{18} \mathrm{~F}$ from the lungs was retarded in aged animals. The accumulation of $5-\left[{ }^{125} I\right]$ iodo-2'-deoxyuridine $\left(\left[{ }^{125} I\right] I U d R\right)$-labeled K1735M2 cells in the lungs of SAMP10 at $24 \mathrm{~h}$ after injection was significantly higher in aged mice. Corresponding to these results, the number of metastatic colonies in the lung was larger in the aged SAMP10 of the experimental tumor metastasis model. The present study demonstrated that the aging process produced a susceptible environment allowing the tumor cells to metastasize due to decrease in the host immune surveillance potential with age.

Key words experimental tumor metastasis; aging; immune surveillance; senescence-accelerated mouse

Since most cancer incidence is related to the accumulation of gene mutations, ${ }^{1)}$ the majority of cancer patients are of the elderly generation and cancer is the highest cause of death for them. These generations are often referred to as "cancer age" and cancer seems to be one of the age-related diseases. ${ }^{2)}$ On the other hand, cancer development is known to depend on the surrounding host environment. ${ }^{3)}$ For example, tumor cell growth in the young is faster than that in the aged due to alterations in host factors. ${ }^{4,5)}$ Thus, it is possible that the aging process also affects cancer development by alteration of the host environment. ${ }^{6,7)}$

Cancer metastasis is often the critical trigger for the death of a patient. It begins to dissociate from primary sites and culminates with the formation of metastatic colonization. ${ }^{8)}$ These processes are very sensitive to the surrounding host factors such as the host resistance to cancerous cells. In a previous study, we suggested that it is a critical step for tumor cells in experimental tumor metastasis to avoid the host immune surveillance associated with immune cells such as natural killer (NK) cells and macrophages.9) Because the early accumulation of metastatic tumor cells in the target organ well reflected the eventual tumor metastasis, it was demonstrated that the immune system functioned to remove the tumor cells from the bloodstream and the target site in the early stage of tumor metastasis. ${ }^{10)}$ From these observations, we speculated that the experimental metastatic potential would be altered with age due to the age-dependent alteration of host resistance to the metastatic tumor cells. In the present study, we investigated the alteration of experimental tumor metastasis between young and aged mice using senescenceaccelerated mice (SAM).

The SAM prone (SAMP) strain was originally established as a mouse model for aging research ${ }^{11,12}$ and shows a more accelerated senescence process than normal mouse, such as a shorter lifespan, a compromised immune system, and development of several geriatric disorders which are frequently observed in aged humans. ${ }^{12,13)}$ Thus, we theorized that aged SAMP was a more appropriate model for cancer research than the young healthy mice usually used in experimental models. We used one of the SAMP strains, SAMP10, which showed several geriatric disorders such as brain atrophy. ${ }^{14,15)}$ In the present study, we investigated the alteration of tumor metastatic potential with age in those mice: 2-month-old SAMP10 was used as the "young" model and an 8-monthold as the "aged". Alteration of the NK activity with age was first examined, because NK cells are well known to work on the first line of defense against mutant cells such as cancerous cells. ${ }^{16,17)}$ Next, we investigated the early accumulation of highly lung-metastatic K1735M2 melanoma cells in the target organ using positron emission tomography (PET) and the $5-\left[{ }^{125} \mathrm{I}\right]$ iodo-2'-deoxyuridine $\left(\left[{ }^{125} \mathrm{I}\right] \mathrm{IUdR}\right)$-labeling method. Finally, we examined experimental tumor metastasis after the intravenous injection of K1735M2 cells in young and aged SAMP10 and evaluated the difference of eventual tumor metastasis with age.

\section{MATERIALS AND METHODS}

Animals Senescence-accelerated mouse prone 10 (SAMP10) was purchased from Japan SLC Inc. (Shizuoka, Japan). Two-month-old SAMP10 was used as young and 8month-old SAMP10 as aged. They rapidly began to show several senescence symptoms such as brain atrophy and dehairing from about 6-months-old, and thus we used 8-monthold SAMP10 mice as an appropriate model for the cancer prone age.

Cells Murine highly lung-metastasic K1735M2 melanoma (K1735M2) cells were originally established by Dr. 
Isaiah J. Fidler at the University of Texas, MD Anderson Cancer Center (TX, U.S.A.) and kindly given us by Dr. Jun Yokota at National Cancer Center (Tokyo, Japan). These cells were cultured in Dulbecco's modified Eagle medium (DMEM) containing 10\% fetal bovine serum (FBS, Japan Bio Serum Co., Ltd., Hiroshima, Japan) at $37^{\circ} \mathrm{C}$ in the presence of $5 \% \mathrm{CO}_{2}$ in a humid atmosphere. NK-sensitive YAC1 cells were purchased from the RIKEN Bioresource Center Cell Bank (Ibaraki, Japan) and cultured in RPMI1640 medium containing $10 \% \mathrm{FBS}$ at $37^{\circ} \mathrm{C}$ in the presence of $5 \%$ $\mathrm{CO}_{2}$

NK Activity Assay To prepare the effector cells for the NK activity assay, young and aged SAMP10 were sacrificed and their spleens sterilely dissected. After mincing the spleen, the splenocytes were carefully flushed several times with an $18 \mathrm{G}$ needle-tipped syringe and passed through nylon mesh ( $41 \mu \mathrm{m}$-pore size) to prepare the singlet cell suspension. The resultant cells were washed twice after removal of erythrocytes, and the number adjusted to $1.25,2.5$, and $5 \times 10^{6}$ cells $/ \mathrm{ml}$. On the other hand, mouse NK-sensitive lymphoma YAC-1 cells were used as the target for ${ }^{51} \mathrm{Cr}$ release assay. The cells were incubated with $\mathrm{Na}_{2}{ }^{51} \mathrm{CrO}_{4}$ solution (GE Healthcare UK, Ltd., England) for $60 \mathrm{~min}$ at $37^{\circ} \mathrm{C}$ to radiolabel with ${ }^{51} \mathrm{Cr}$. After removal of free ${ }^{51} \mathrm{Cr}$, these cells were resuspended at a concentration of $1 \times 10^{5}$ cells $/ \mathrm{ml}$. The NK activity of each mouse was measured by ${ }^{51} \mathrm{Cr}$ release assay as follows: Both the effector and the target cells were added to 96-well plates at the ratio of effector to target $(\mathrm{E} / \mathrm{T})$ of $50: 1$, $25: 1$, and $12.5: 1$, and incubated for $4 \mathrm{~h}$ at $37^{\circ} \mathrm{C}$. After centrifugation of the cell suspension, radioactivity released into the supernatant was determined by a gamma counter. The spontaneous release and the maximum release were determined by adding RPMI medium containing 10\% FBS or $0.1 \%$ TritonX-100 solution to the target cell suspension, respectively. The specific cytotoxicity of NK cells was determined using the following formula:

$$
\% \text { lysis }=\frac{\text { experimental release }- \text { spontaneous release }}{\text { maximum release }- \text { spontaneous release }} \times 100
$$

Positron Emission Tomography (PET) Study $\mathrm{K} 1735 \mathrm{M} 2$ cells were radiolabeled with $\left[2-{ }^{18} \mathrm{~F}\right] 2$-fluoro-2deoxy-D-glucose ( $\left[{ }^{18} \mathrm{~F}\right] \mathrm{FDG}$, Hamamatsu Photonics K.K., Shizuoka, Japan) as described previously. ${ }^{18)}$ In brief, they were incubated with $\left[{ }^{18} \mathrm{~F}\right] \mathrm{FDG}$ solution in the presence of phosphate-buffered saline containing $\mathrm{Ca}^{2+}$ and $\mathrm{Mg}^{2+}$ $[\operatorname{PBS}(+)]$ for $30 \mathrm{~min}$ at $37^{\circ} \mathrm{C}$. Then, $\left[{ }^{18} \mathrm{~F}\right] \mathrm{FDG}$-labeled $\mathrm{K} 1735 \mathrm{M} 2$ cells were collected in the presence of EDTA and centrifuged to remove unlabeled $\left[{ }^{18} \mathrm{~F}\right] \mathrm{FDG}$. The cells were passed through the nylon mesh ( $41 \mu \mathrm{m}$-pore size) to obtain singlet cells and the number adjusted to $1 \times 10^{4}$ cells/mouse for injection. PET study was started immediately after intravenous injection of the cells into young and aged SAMP10 via a tail vein and the image was scanned for $120 \mathrm{~min}$ with a SHR-7700 PET-camera (Hamamatsu Photonics K.K., Shizuoka, Japan). The accumulated image in the lung every $15 \mathrm{~min}$ and the time-activity curves obtained from the mean pixel radioactivity in the region of interest (ROI) of the PET images were used to evaluate the accumulation of tumor cells in the lung. After the PET scan, the mice were sacrificed under anesthesia with diethylether and the biodistribution of ${ }^{18} \mathrm{~F}$-labeled cells was confirmed with a gamma counter.
Distribution of Metastatic Tumor Cells K1735M2 cells were radiolabeled with $5-\left[{ }^{125} \mathrm{I}\right]$ iodo- $2^{\prime}$-deoxyuridine ([ $\left.{ }^{125} \mathrm{I}\right] \mathrm{IUdR}$, GE Healthcare UK, Ltd.,) as described. $\left.{ }^{19}\right)$ Briefly, these cells were incubated overnight with $\left[{ }^{125} \mathrm{I}\right] \mathrm{IUdR}$ at $37^{\circ} \mathrm{C}$ in the presence of $5 \% \mathrm{CO}_{2}$. After the incubation, the cells were collected and centrifuged to remove the unincorporated $\left[{ }^{125} \mathrm{I}\right] \mathrm{IUdR}$ and the number adjusted to $1 \times 10^{4}$ cells/mouse for injection. These cells were intravenously injected into young and aged mice. At $24 \mathrm{~h}$ after the injection, each mouse was sacrificed under anesthesia with diethylether to collect the blood and organ (heart, lung, liver, spleen, and kidney) samples. The accumulation of tumor cells in each organ was determined by the radioactivity. Distribution data was presented as $\%$ injected dose per tissue. The total weight of blood was assumed to be $7.56 \%$ of the body weight.

Experimental Lung Metastatic Model K1735M2 cells $\left(5 \times 10^{4}\right.$ cells/mouse) were intravenously injected into each mouse via a tail vein and allowed to form metastatic colonies in their lungs. Twenty-one days after injection of the tumor cells, the animals were sacrificed under diethylether anesthesia, and their perfused lungs were isolated. The tumor metastasis was evaluated by counting the number of metastatic colonies on the surface of the lungs.

Statistic Analysis The variance in a group was evaluated by $F$-test, and the differences among the mean number of metastatic colonies were evaluated by Student's $t$-test.

\section{RESULTS}

NK Activity in Aged SAMP10 In the previous study, we suggested that the metastatic tumor cells interacted with immune cells such as NK cells and macrophages in the early stage of experimental tumor metastasis, and that avoidance of their immune surveillance was critical for the completion of tumor metastasis. ${ }^{9)}$ Thus, we predicted that the process of aging might enhance the tumor metastasis because it would decrease the host immune surveillance potential. Therefore, we first investigated the NK activity in young and aged mice as an index of the immune surveillance potential. SAMP10 was used as an aged model in this study because it showed senescence characteristics in less than 1 year. ${ }^{12)}$ As a result, the NK activity of aged SAMP10 was significantly decreased in comparison to that of young mice (Fig. 1). This NK activ-

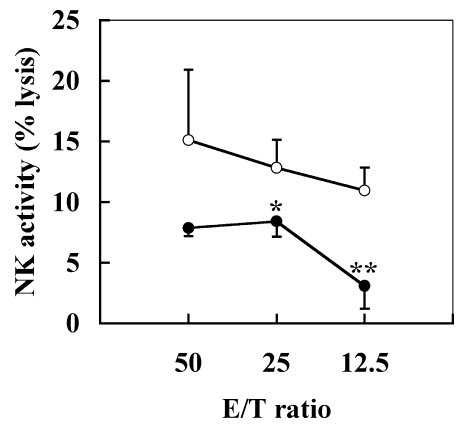

Fig. 1. Decrease of NK Activity with Age in SAMP10

Graph shows the cytotoxicity of NK cells in the spleen (E: effector cells) of young (O) and aged (O) SAMP10 against ${ }^{51} \mathrm{Cr}$-labeled YAC-1 target cells (T: target cells). The radioactivity released into supernatant medium was measured by a gamma counter and the NK activity was determined as the formula described in Materials and Methods. Significant differences from "young" are indicated $(* p<0.05 ; * * p<0.01)$. Similar results were obtained in a separate experiment. 


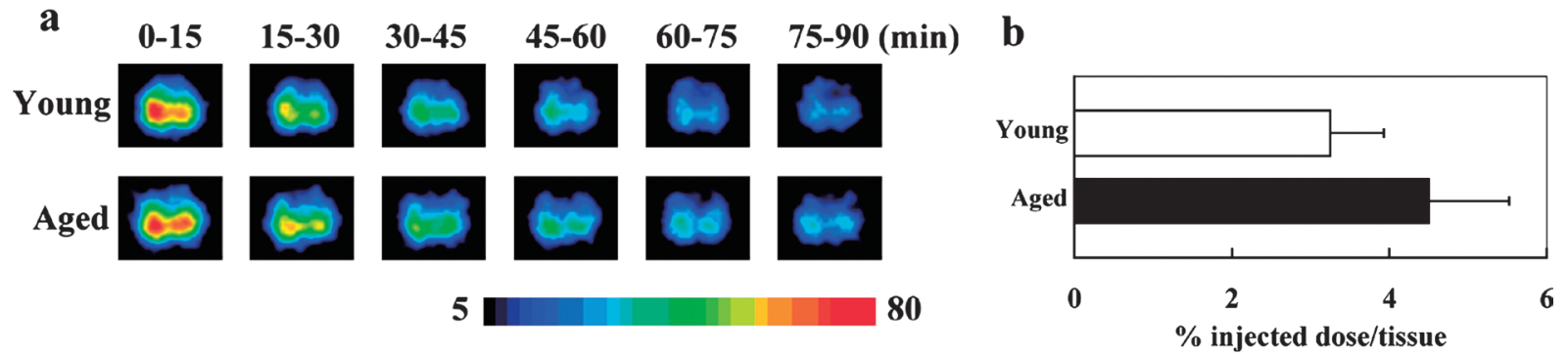

Fig. 2. PET Analysis of Tumor Cell Trafficking in SAMP10

$\left[{ }^{18} \mathrm{~F}\right] \mathrm{FDG}-\mathrm{labeled} \mathrm{K} 1735 \mathrm{M} 2$ cells $\left(1 \times 10^{4}\right.$ cells/mouse $)$ were intravenously injected into young and aged SAMP10. PET images represent 15 min accumulation changes in the lung of each mouse (a), and the accumulation of these cells in the lung $120 \mathrm{~min}$ after injection is shown in the graph (b). PET analyses were performed thrice, and similar results were obtained.

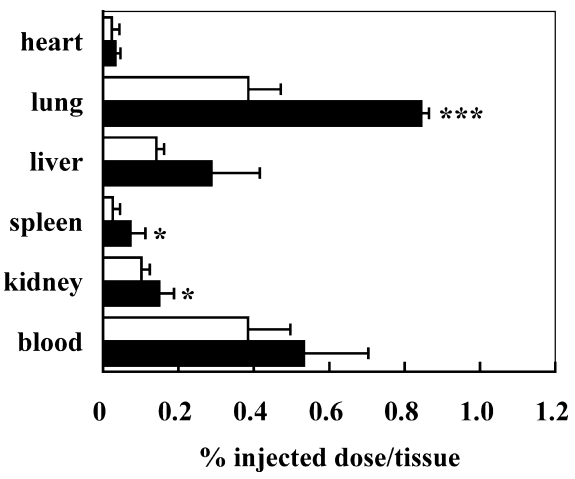

Fig. 3. Distribution of $\left[{ }^{125} \mathrm{I}\right] \mathrm{IU} d \mathrm{R}-\mathrm{Labeled} \mathrm{K1735 \textrm {M } 2}$ Melanoma Cells in SAMP10

$\left[{ }^{125} \mathrm{I}\right] \mathrm{IUdR}$-labeled K1735M2 melanoma cells $\left(1 \times 10^{4}\right.$ cells/mouse $)$ were injected into young (open column) and aged (closed column) SAMP10. The columns indicate the percent accumulation of $\left.{ }^{125} \mathrm{I}\right] \mathrm{IUdR}$-labeled K1735M2 melanoma cells $24 \mathrm{~h}$ after injection. Significant differences from "young" are indicated $(* p<0.05 ; * * * p<0.001)$. Similar results were obtained in a separate experiment.

ity was dependent on the number of effector cells which were the spleen cells sacrificed from each mouse, and the decrease in NK activity of aged mice was observed at every $\mathrm{E} / \mathrm{T}$ ratio (Fig. 1). This result suggested that the immune surveillance potential of SAMP10 was decreased in an age-dependent manner.

PET Analysis of Metastatic Tumor Trafficking in SAMP10 To investigate the difference in tumor cell trafficking between ages, we performed the PET analysis to observe the early stage of tumor metastasis non-invasively in real time. The previous study indicated that the early accumulation of metastatic tumor cells in the target organ strongly foretold the eventual tumor metastasis in experimental metastatic models. ${ }^{9,18)}$ The accumulation of $\left[{ }^{18} \mathrm{~F}\right]$ FDG-labeled K1735M2 cells in the lung in young and aged mice was observed: Lung is known as the target organ for K1735M2 cells after their injection into the bloodstream. Figure $2 \mathrm{a}$ shows the accumulated image every $15 \mathrm{~min}$ in the lung, and it is apparent that the tumor cells gradually began to remain in the lung of aged mice more than in that of young mice $90 \mathrm{~min}$ after injection. Actually, the subsequent accumulation of K1735M2 cells 120 min after injection was higher in aged mice (Fig. 2b). These results suggested that the aged mice tended to fail to remove metastatic tumor cells from the target organ in the very early stage of metastasis, perhaps due to the decreased immune potential in these mice.

Distribution of K1735M2 Cells in SAMP10 PET

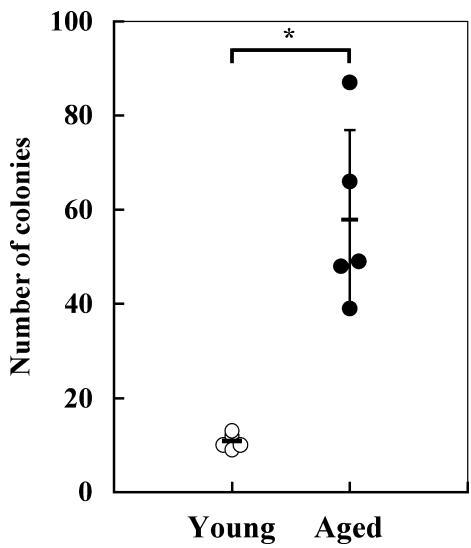

Fig. 4. Impaired Experimental Lung Metastasis with Age

K1735M2 cells $\left(5 \times 10^{4}\right.$ cells/mouse) were intravenously injected into young and aged SAMP10 and the metastatic colonies in the lung were counted on day 21 after tumor implantation. The circles in the graph show the number of colonies in individual mice and black bars show the average number of colonies in each mouse. Asterisks indicate the significant differences $(* p<0.05)$. Similar results were obtained in a separate experiment.

analysis is suitable for the investigation of very early trafficking, usually up to $2 \mathrm{~h}$, of metastatic tumor cells. On the other hand, a biodistribution study using $\left[{ }^{125} \mathrm{I}\right] \mathrm{IUdR}$-labeled cells enables examination of the distribution of these cells up to several days, although it is an invasive method. Therefore, we next examined the further distribution of tumor cells using the $\left[{ }^{125} \mathrm{I}\right] \mathrm{IU} d \mathrm{R}$-labeling method. $\left[{ }^{125} \mathrm{I}\right] \mathrm{IU} d \mathrm{R}$-labeled K1735M2 cells were intravenously injected into young and aged mice, and their accumulation in the lung $24 \mathrm{~h}$ later was measured: the $24 \mathrm{~h}$-accumulation of these cells in the lung was significantly higher in aged mice than that in young mice (Fig. 3). This result also indicated that the cell accumulation in the lung after the intravenous injection of K1735M2 cells was impaired by age and reflected the PET result.

Age-Dependent Alteration of Experimental Tumor Metastasis in SAMP10 Finally, experimental tumor metastasis after injection of metastatic tumor cells into the bloodstream was investigated. K1735M2 cells were intravenously injected into young and aged SAMP10, and they were allowed to form metastatic colonies in the lung. Intravenous injection of $5 \times 10^{4} \mathrm{~K} 1735 \mathrm{M} 2$ cells resulted in the formation of some metastatic colonies in the lung of aged mice whereas those in young mice were rarely observed (Fig. 4). This result indicated that the experimental lung metastasis occurred significantly in aged SAMP10, suggesting that the process of aging might enhance the tumor metastasis. 


\section{DISCUSSION}

Aging occurs to all of us and brings various alterations in a body. In particular, the process of aging brings the alteration of homeostasis including the decrease of various functions in organs. ${ }^{20,21)}$ Actually, aged people tend to assimilate diseases easier. Cancer often develops in middle age or later except the particular type of cancer such as leukemia, meaning that cancer development is definitely affected by the change of host environment with age. ${ }^{22,23)}$ Despite these statistics, very young animals have been widely used for basal cancer studies such as the evaluation of cancer therapy and cancer prevention, since it takes a long time to prepare aged animals. In the present study, we investigated whether experimental tumor metastasis was altered with age. We used 8month-old SAMP10 as a model for cancer age humans, since this model showed several accelerated senescence processes such as dehairing and brain atrophy. ${ }^{12)}$ It is still not clear why SAMP acquires these characteristics, however, they do adequately reflect human aging. ${ }^{24)}$

In blood-borne metastasis, it is generally known that a certain number of metastatic tumor cells is required for the establishment of a metastatic focus, since a small number of tumor cells might be removed by the host immune defense system. In fact, we previously observed that the injection of a small number $\left(1 \times 10^{4}\right)$ of lung-metastatic B16BL6 cells or liver-metastatic RAW117 cells did not create metastatic colonies. ${ }^{9}$ PET analysis of the metastatic tumor cell trafficking revealed that retention of the metastatic cells at the target sites was lower when a lower number of cells was injected into the bloodstream. ${ }^{18)}$ Furthermore, in mice pretreated with 2-chloroadenosine, which can exhibit a lethal effect on mouse NK cells and macrophages, the early accumulation of tumor cells at the metastatic organ and actual tumor metastasis were observed, indicating both the importance of immune surveillance at the early phase of metastasis and that this event had adequate impact on actual tumor metastasis. ${ }^{\text {) }}$

In this study, we first compared the potential of the immune surveillance system between young and aged SAMP10: The cytotoxic effect of splenocytes derived from both groups against NK-sensitive YAC-1 cells was investigated. In particular, NK cells as well as macrophages are generally known to associate with innate immunity, especially immune surveillance, and to exist as the first line of defense against exogenous agents and mutated cells such as cancerous cells. ${ }^{10,25)}$ There have been trials using NK cells for cancer immunotherapy because of their strong and specific activity against cancerous cells. ${ }^{25)}$ As shown in Fig. 1, we found a decrease in NK activity in aged mice compared with young ones. Since aging is thought to relate to the accumulation of oxidative stress, ${ }^{3,26)}$ it is possible that an age-dependent burden of oxidative stress causes degradation of the immune defense system against the tumor cells. In fact, NK cells are known to be quite sensitive to oxidative stress and their activity is often affected by reactive oxygen species. ${ }^{27)}$ This evidence supports the idea that the aging process produces a susceptible environment for tumor cells in the bloodstream to metastasize. Secondly, to elucidate the differences of tumor metastasis between young and aged SAMP10, we used PET to analyze the trafficking of metastatic tumor cells non-invasively in real time. PET analysis indicated that $\left[{ }^{18} \mathrm{~F}\right]$ FDG-labeled K1735M2 cells tended to remain in the lung of aged mice, whereas they rapidly disappeared in that of young mice (Figs. 2a, b). Furthermore, $\left.{ }^{[25} \mathrm{I}\right] \mathrm{IUdR}$-labeled K1735M2 cells significantly remained in the lung of aged mice $24 \mathrm{~h}$ after tumor cell injection (Fig. 3). These data suggested that, in aged mice, the tumor cells injected into the bloodstream began to accumulate in the target organ at an early phase of experimental metastasis, although in young mice these cells were removed from that organ. When SAMP10 were pretreated with 2-chloroadenosine, the accumulation of $\left.{ }^{125} \mathrm{I}\right] \mathrm{IUdR}$-labeled $\mathrm{K} 1735 \mathrm{M} 2$ cells in the lung was enhanced (data not shown). Finally, we performed an experimental lung metastasis assay in young and aged SAMP10 and found that a larger number of metastatic colonies of these cells at the lung was observed in aged mice (Fig. 4). Furthermore, when 13-month-old SAMP10 mice were injected with the same number of K1735M2 cells, many more metastatic colonies were observed than in 8month-old mice (data not shown). These observations indicated that aging facilitates the formation of metastatic colonies in the target organ after cell injection into the bloodstream. Spontaneous tumor metastasis, however, is processed through various steps and the experimental tumor metastatic model used in this study mimics only part of the process. The characteristic of tumor cells in aged animals is known to be different from that of the cultured cells and the former generally shows their senescence. ${ }^{20,22)}$ Furthermore, it is reported that solid tumor growth in aged mice is different from that in young mice: it is more malignant in young mice because of the alteration of the surrounding environment and the subsequent angiogenesis. ${ }^{28-30)}$ It is uncertain whether spontaneous tumor metastasis is impaired or enhanced with aging. However, in this study, our findings suggested that tumor metastasis was enhanced in aged animals once the tumor cells had come into the bloodstream.

In conclusion, we demonstrated that aging produced a susceptible environment for metastatic tumor cells in the bloodstream to complete metastasis, and one of the reasons it occurs is due to the decreased immune surveillance potential with age. Since the responses of aged animals are different from those of young ones, the use of an aged animal model including SAMP in such cancer studies as therapeutic and for preventive evaluation may reveal more predictable results for actual human cancers.

Acknowledgments The authors thank Dr. Takeharu Kakiuchi and Mr. Hiroyuki Oba at Hamamatsu Photonics K.K. for technical assistance in the PET study, Dr. Jun Yokota at the National Cancer Center for giving the K1735M2 melanoma cells, and Dr. Keiko Unno and all members in the Department of Medical Biochemistry at the University of Shizuoka for helpful advice. This work was supported in part by a Grant-in-Aid for Scientific Research from the Japan Society for the Promotion of Science.

\section{REFERENCES}

1) Hahn W. C., Weinberg R. A., N. Engl. J. Med., 347, 1593-1603 (2002).

2) DePinho R. A., Nature (London), 408, 248-254 (2000).

3) Anisimov V. N., Cancer Control, 14, 23-31 (2007).

4) Pili R., Guo Y., Chang J., Nakanishi H., Martin G. R., Passaniti A., J. 
Natl. Cancer Inst., 86, 1303-1314 (1994).

5) Maffini M. V., Calabro J. M., Soto A. M., Sonnenschein C., Am. J. Pathol., 167, 1405-1410 (2005).

6) Anisimov V. N., Crit. Rev. Oncol. Hematol., 45, 277-304 (2003).

7) Krtolica A., Campisi J., Int. J. Biochem. Cell Biol., 34, 1401-1414 (2002).

8) Mundy G. R., Nat. Rev. Cancer, 2, 584-593 (2002).

9) Kikkawa H., Imafuku H., Tsukada H., Oku N., FEBS Lett., 467, 211$216(2000)$.

10) Wallace M. E., Smyth M. J., Springer Semin. Immunopathol., 27, 4964 (2005).

11) Takeda T., Matsushita T., Kurozumi M., Takemura K., Higuchi K., Hosokawa M., Exp. Gerontol., 32, 117-127 (1997).

12) Takeda T., Hosokawa M., Higuchi K., Exp. Gerontol., 32, 105-109 (1997).

13) Takeda T., Neurobiol. Aging, 20, 105-110 (1999).

14) Miyamoto M., Exp. Gerontol., 32, 139-148 (1997).

15) Kawamata T., Akiguchi I., Maeda K., Tanaka C., Higuchi K., Hosokawa M., Takeda T., Microsc. Res. Tech., 43, 59-67 (1998).

16) Page G. G., Ben-Eliyahu S., Neuroimmunomodulation, 7, 160-168 (2000).

17) Farag S. S., Fehniger T. A., Becknell B., Blaser B. W., Caligiuri M. A., Expert Opin. Biol. Ther, 3, 237-250 (2003).

18) Kikkawa H., Tsukada H., Oku N., Cancer, 89, 1626-1633 (2000).
19) Koike C., Watanabe M., Oku N., Tsukada H., Irimura T., Okada S. Cancer Res., 57, 3612-3619 (1997).

20) Herbig U., Ferreira M., Condel L., Carey D., Sedivy J. M., Science, 311, 1257 (2006).

21) Bavik C., Coleman I., Dean J. P., Knudsen B., Plymate S., Nelson P. S., Cancer Res., 66, 794-802 (2006).

22) Campisi J., Cell, 120, 513-522 (2005).

23) Campisi J., Nat. Rev. Cancer, 3, 339-349 (2003).

24) Carter T. A., Greenhall J. A., Yoshida S., Fuchs S., Helton R., Swaroop A., Lockhart D. J., Barlow C., Genome Biol., 6, R48.1-R48.17 (2005).

$25)$ Smyth M. J., Hayakawa Y., Takeda K., Yagita H., Nat. Rev. Cancer, 2 , $850-861$ (2002).

26) Mori A., Utsumi K., Liu J., Hosokawa M., Ann. N.Y. Acad. Sci., 854, $239-250$ (1998).

27) Nakamura K., Matsunaga K., Cancer Biother. Radiopharm., 13, $275-$ 290 (1998).

28) Rivard A., Fabre J. E., Silver M., Chen D., Murohara T., Kearney M., Magner M., Asahara T., Isner J. M., Circulation, 99, 111-120 (1999).

29) Rivard A., Berthou-Soulie L., Principe N., Kearney M., Curry C., Branellec D., Semenza G. L., Isner J. M., J. Biol. Chem., 275, $29643-$ 29647 (2000).

30) Coppe J. P., Kauser K., Campisi J., Beausejour C. M., J. Biol. Chem., 281, 29568-29574 (2006). 